\title{
Distinct Mutation Patterns Reveal Melanoma Subtypes and Influence Immunotherapy Response in Advanced Melanoma Patients
}

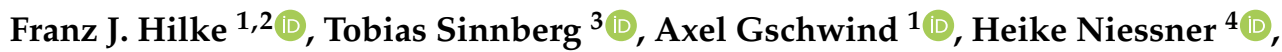 \\ German Demidov ${ }^{1}{ }^{(}$, Teresa Amaral ${ }^{4,5}{ }^{\infty}$, Stephan Ossowski $\left.{ }^{1,6}{ }^{(}\right)$, Irina Bonzheim ${ }^{7}$, \\ Martin Röcken ${ }^{4}$, Olaf Riess ${ }^{1,6}$, Claus Garbe ${ }^{4}$, Christopher Schroeder ${ }^{1, *}$ and Andrea Forschner 4 \\ 1 Institute of Medical Genetics and Applied Genomics, University Hospital Tübingen, 72076 Tübingen, \\ Germany; franz.hilke@charite.de (F.J.H.); axel.gschwind@uni-tuebingen.de (A.G.); \\ german.demidoc@uni-tuebingen.de (G.D.); stephan.ossowski@med.uni-tuebingen.de (S.O.); \\ olaf.riess@med.uni-tuebingen.de (O.R.) \\ 2 Derpartment of Dermatology, Venerology and Allergology, Charité-Universitätsmedizin Berlin, \\ 10117 Berlin, Germany \\ 3 Image-Guided and Functionally Instructed Tumor Therapies (iFIT) Cluster of Excellence (EXC 2180), \\ University of Tübingen, 72076 Tübingen, Germany; tobias.sinnberg@med.uni-tuebingen.de \\ 4 Department of Dermatology, University Hospital Tübingen, 72076 Tübingen, Germany; \\ heike.niessner@med.uni-tuebingen.de (H.N.); teresa.amaral@med.uni-tuebingen.de (T.A.); \\ martin.roecken@med.uni-tuebingen.de (M.R.); claus.garbe@med.uni-tuebingen.de (C.G.); \\ andrea.forschner@med.uni-tuebingen.de (A.F.) \\ 5 Portuguese Air Force Health Care Direction, 1649-020 Lisbon, Portugal \\ 6 German DFG NGS Competence Center, NCCT, 72076 Tübingen, Germany \\ 7 Institute of Pathology and Neuropathology, University Hospital Tübingen, 72076 Tübingen, Germany; \\ irina.bonzheim@med.uni-tuebingen.de \\ * Correspondence: christopher.schroeder@med.uni-tuebingen.de; Tel.: +49-7071-29-72296
}

Received: 28 July 2020; Accepted: 16 August 2020; Published: 20 August 2020

\begin{abstract}
The detection of somatic driver mutations by next-generation sequencing (NGS) is becoming increasingly important in the care of advanced melanoma patients. In our study, we evaluated the NGS results of 82 melanoma patients from clinical routine in 2017. Besides determining the tumor mutational burden (TMB) and annotation of all genetic driver alterations, we investigated their potential as a predictor for resistance to immune checkpoint inhibitors (ICI) and as a distinguishing feature between melanoma subtypes. Melanomas of unknown primary had a similar mutation pattern and TMB to cutaneous melanoma, which hints at its cutaneous origin. Besides the typical hotspot mutation in $B R A F$ and $N R A S$, we frequently observed CDKN2A deletions. Acral and mucosal melanomas were dominated by CNV alterations affecting PDGFRA, KIT, CDK4, RICTOR, CCND2 and CHEK2. Uveal melanoma often had somatic SNVs in GNA11/Q and amplification of MYC in all cases. A significantly higher incidence of BRAF V600 mutations and EGFR amplifications, PTEN and TP53 deletions was found in patients with disease progression while on ICI. Thus, NGS might help to characterize melanoma subtypes more precisely and to identify possible resistance mechanisms to ICI therapy. Nevertheless, NGS based studies, including larger cohorts, are needed to support potential genetic ICI resistance mechanisms.
\end{abstract}

Keywords: Genome of advanced melanoma; acral; mucosal; uveal; melanoma of unknown origin; tumor mutation burden; TMB; immune checkpoint inhibitors; next-generation sequencing 


\section{Introduction}

In the last decade, multiple large-scale sequencing studies have elucidated the genetic landscape of cutaneous melanoma and led to the classification of the genetic subtypes BRAF mutant, RAS mutant, NF1 mutant or triple wild-type melanoma [1]. In conjunction with earlier exome sequencing studies [2,3], it was shown that the RTK, RAS/RAF/ERK, PI3K and cell cycle pathways were the significant drivers for the oncogenesis of cutaneous melanoma. Additionally, these comprehensive sequencing studies have revealed the genetic landscape not only for cutaneous melanoma but also for acral and mucosal melanoma [4], resulting in the identification of further driver alterations and highlighting the impact of copy number changes in these subtypes $[5,6]$.

Beyond the genetic characterization, the identification of predictive and prognostic markers for treatment responses became a primary focus over the past years $[7,8]$. Especially in the light of treatment failure in patients treated with immunotherapy, certain genes and signaling cascades, like an amplification of MDM2 and EGFR [9-11], PTEN deletions and loss of functions mutations in $J A K 2$ or the interferon gamma signaling were identified as potential markers of primary or acquired resistance [12-15]. Also, the recent work on the resistance mechanisms in melanoma patients treated with $B R A F$ or $M E K$ inhibitor therapy revealed new therapeutic strategies $[7,8,16]$.

Therefore, we analyzed the results of next-generation sequencing of 82 patients with advanced melanoma, whose primary tumors or metastases had been sequenced as part of routine clinical care in 2017. We display the diversity of genetic mutations and affected pathways among this clinical cohort, highlighting both genetic differences and similarities among the melanoma subtypes and show potential genetic resistance mechanisms to ICI. We aimed at identifying mutational patterns revealing the origin of melanomas of unknown primary or predicting response to ICI.

\section{Materials and Methods}

\subsection{Patients and Tumor Tissue}

We included all melanoma patients of our Center for Dermatooncology, whose tumors had been sequenced in 2017 as part of routine clinical care. All patients had been informed in a personal conversation with their dermato-oncologist and human geneticist about the procedure of tumor sequencing. Written consent was obtained from all patients. Clinical data were obtained from the patients' records. All patients had given their consent for their data to be made available for research. The local ethics committee of the University Tuebingen approved this study (approval number 103/2018BO2). The formalin-fixed paraffin-embedded tissue used for sequencing consisted of the latest available tissue, usually metastases that had been removed recently. Genomic DNA was extracted from macrodissected $5 \mu \mathrm{m}$ paraffin sections using the Maxwell ${ }^{\circledR}$ RSC DNA FFPE Kit and the Maxwell ${ }^{\circledR}$ RSC Instrument (Promega, Madison, WI, USA) according to the manufacturer's instructions.

\subsection{Sequencing}

The sequencing panel was applied in three different versions, targeting 336 cancer-associated genes in the primary setting (ssSCv2; Table S1), 677 (ssSCv3; Table S2) or 693 (ssSCv4; Table S3) genes as well as intronic regions for the detection of distinct fusions in the updated versions (Table S4). As the sequencing panel changed in the course of this study, we focused the analysis on the 275 common genes present in all versions. All samples were sequenced on an Illumina device (e.g., NextSeq500, Illumina Inc., San Diego, CA, USA). Data processing was done by an in-house analysis pipeline (megSAP, https://github.com/imgag/megSAP) using the open source tool BWA for mapping [17] and strelka2 for somatic variant detection [18]. The detection of somatic copy number alterations was done by our in-house software tool clinCNV [19]. Variants were annotated using SnpEff / SnpSift [20,21] and the publicly available Cancer Genome Interpreter [22] for information about driver alterations. The R package Maftools version 2.0.16 was used for visualization [23]. 


\subsection{Tumor Mutational Burden}

Tumor mutation burden (TMB) was calculated as the number of all somatic alterations (coding SNVs and INDELs) based on the target size of the used panel. Since all three panel versions are designed to detect driver mutations in known tumor suppressor and oncogenes, we had to adjust the calculation to avoid overrating the TMB. The Formula is:

$$
\mathrm{TMB}=\left[\frac{\left(\frac{\text { Somatic }- \text { Known_Tumorgenes }}{\text { Target size }} \times \text { Genome size }\right)+\text { Tumorgenes }}{\text { Genome size }}\right]
$$

\subsection{Gene Set Enrichment Analysis}

The gene set enrichment was performed using the clinical feature histopathological subtype associated with the samples. The enrichment analysis was done using the gene set enrichment function of the $\mathrm{R}$ package MAFTOOLs Version 2.0.16 [23]. It performs various groupwise and pairwise comparisons to identify enriched mutations for every category within a clinical feature.

\subsection{Statistics}

Statistical analysis was performed using the statistical program for social sciences SPSS version 25 (IBM, Edicott, NY, USA) as well as Graphpad Prism Version 8.3.0 (GraphPad Software, LLC, San Diego, CA, USA). The distribution of TMB, SNVs and CNVs was compared between the different melanoma types using Mann-Whitney U test. The overall survival was calculated using the overall survival function in GraphPad Prism Version 8. Follow-up time was defined as the time between the initiation of immune checkpoint inhibition (ICI) and the date of the last follow-up or death from any cause.

\section{Results}

In total, 82 tumor tissue samples from 82 different patients were sequenced and analyzed. The cohort included patients with cutaneous melanoma $(n=42)$, acral $(n=14)$, mucosal $(n=9)$ and uveal melanoma $(n=8)$, as well as melanoma of unknown primary $(n=9)$. The gender distribution was almost equal, with $37(45 \%)$ females and $45(55 \%)$ males. The median age at the time of primary melanoma diagnosis was 57 years (IQR 46-67 years). The majority of patients $(n=75)$ had been treated with ICI, either with the PD-1 antibodies nivolumab and pembrolizumab alone and/or nivolumab in combination with ipilimumab, a CTLA-4 antibody (Figure 1, Table 1).

Table 1. Clinical characteristics of the 82 patients.

\begin{tabular}{lc}
\hline Patient Characteristics & \\
\hline Age at the first diagnosis of melanoma & years \\
Median & $\mathbf{5 7}(\mathbf{4 6 - 6 7 )}$ \\
Range & $\mathbf{1 7 - 8 5}$ \\
\hline Sex & no of patients (\%) \\
Female & $37(45)$ \\
Male & $45(55)$ \\
\hline Melanoma type & no. of patients (\%) \\
Cutaneous & $42(51)$ \\
Acral & $14(17)$ \\
Uveal & $8(10)$ \\
Mucosal & $9(11)$ \\
Occult & $9(11)$ \\
\hline Tumor thickness of primary melanoma & mm \\
Median (IQR) & $3.3(1.8-5.1)$ \\
Range & $0.38-5.1$ \\
\hline
\end{tabular}


Table 1. Cont.

\begin{tabular}{lc}
\hline Patient Characteristics & \\
\hline The tumor stage at the time of tumor sequencing & no. of patients (\%) \\
Stage II & $5(6)$ \\
Stage IV & $77(94)$ \\
Immunotherapy & $76 / 82(93)$ \\
Targeted therapy & $21(26)$ \\
Chemotherapy & $4(5)$ \\
No systemic treatment & $5(6)$ \\
\hline Origin of the tissue sequenced & no of patients (\%) \\
Lymph node & $19(23)$ \\
Other metastasis & $57(70)$ \\
Primary melanoma & $6(7)$ \\
\hline Pretreatment of tissue sequenced & no. of patients (\%) \\
Tissue therapy naïve & $52(63)$ \\
Tissue progressive under ICI & $18(22)$ \\
Tissue progressive under targeted therapy & $8(10)$ \\
Tissue progressive under chemotherapy & $4(5)$ \\
\hline
\end{tabular}

\subsection{Driver Alterations and Signaling Cascade}

Comprehensive sequencing of the 82 tumor samples identified in total 1650 somatic variants (SNVs and INDELs) and 2.137 somatic copy number alterations, such as amplification or deletion of a complete gene (Tables S5 and S6). We obtained a median number of $10 \mathrm{SNVs} / \mathrm{INDELs} \mathrm{per} \mathrm{patient} \mathrm{and} \mathrm{a} \mathrm{median}$ TMB of 5.82 (range: 0-151.75; Table S7). Furthermore, we referred to CancerGenomeInterpreter.org for the annotation of driver mutations and revealed a total number of 527 driver alterations, 283 SNVs and 244 CNVs, affecting 149 genes (supplement Tables S8 and S9).

The genomic classification of our cohort into BRAF-, RAS-, NF1-mutated or triple-wildtype showed that more than half of the patients carried a driver mutation in either one of the three genes (Figure $1 \mathrm{~b}$ ). The serine/threonine kinase $B R A F$ was the most frequently mutated gene of this cohort (28\%). The vast majority were hotspot mutations V600E/K/R (91\%). Also, one patient had the BRAF G466E driver mutation, and another had two simultaneous driver mutations at positions P367S and K601E. The second most frequent mutation was the GTPase NRAS (24\%) with Q61K/L/R mutations. In two patients, we observed the rare combination of simultaneously occurring hotspot mutations in the genes BRAF (V600E) and NRAS (Q61K and R, respectively). Moreover, hotspot mutations in HRAS (Q61R) and KRAS (G12D, D119H) were present in one, two patients, respectively (not shown in Figure 1). Driver mutations in the gene NF1 were rare with $6 \%$, while three of the patients either had a $B R A F$ or NRAS hotspot mutation. The rest of the cohort, 36 patients $(44 \%)$, were classified as triple wild-type. Besides driver mutations in the genes $B R A F, N R A S$ and NF1, we found frequent activating amplifications in the receptor tyrosine kinases ERBB3 and MET, as well as in the signal transducers KRAS and PTPRD (Figure 1c). Thus, the RTK/RAS/MAP kinase signaling pathway (hereafter RTK/RAS) is most frequently affected by driver mutations in our cohort (79\%). Interestingly, the activating mutations in $E R B B 3, M E T$ and $K R A S$ were more abundant in the triple wild-type group.

The genes most frequently affected by copy number alterations (deletion or amplification) were CDKN2A (24\%) and CCND3 (23\%), both of which belong to the cell cycle control (Figure 1c). Activating MYC amplifications were also detected in $24 \%$ of the patients. The PI3K signaling cascade was the third most frequent mutated signaling pathway (38\%) with driver mutations in the genes PTEN (17\%) and RICTOR (12\%). Furthermore, we found frequent driver mutations in the Wnt and SWI/SNF signaling pathway (CTNNB1, ARID2, and SMARCA4). 
(a)

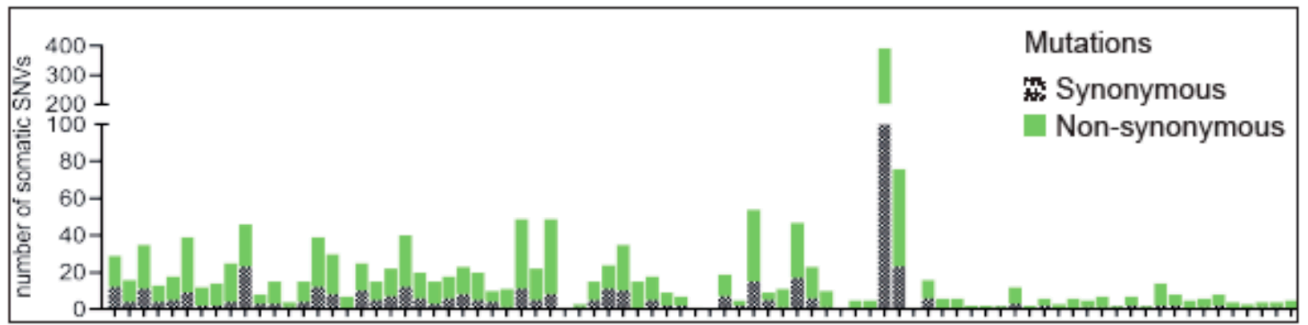

(b)
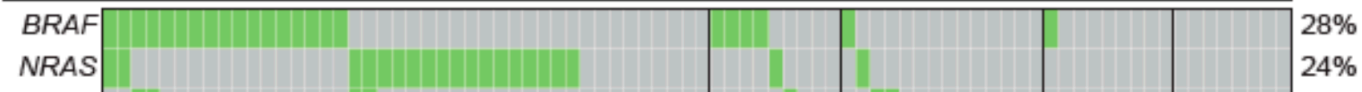

\begin{tabular}{l|l|l|l|l|} 
& \\
\hline
\end{tabular}

\begin{tabular}{l|l|l|l|} 
PTEN & $9 \%$
\end{tabular}

\begin{tabular}{l|l|l|l|} 
CTNNB1 & $7 \%$
\end{tabular}

GNA11 $1107 \%$

\begin{tabular}{l|l|l|l|l|l|l|} 
& $6 \%$
\end{tabular}

\begin{tabular}{l|l|l|l|l|} 
ARID2 & $6 \%$
\end{tabular}

\begin{tabular}{l|l|l|l|} 
FGFR2 & $6 \%$
\end{tabular}

\begin{tabular}{l|l|l|l}
$N F 1$ & $6 \%$
\end{tabular}

NOTCH4

\begin{tabular}{l|l|l|l|} 
SF3B1 & $6 \%$
\end{tabular}

\begin{tabular}{l|l|l} 
IKZF1 & $5 \%$
\end{tabular}

SMARCA4 DI D D

\begin{tabular}{l|l|l|l|} 
GNAQ & & $4 \%$
\end{tabular}

\begin{tabular}{l|l|l|l|} 
BAP1 & $4 \%$
\end{tabular}

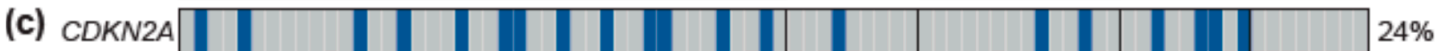

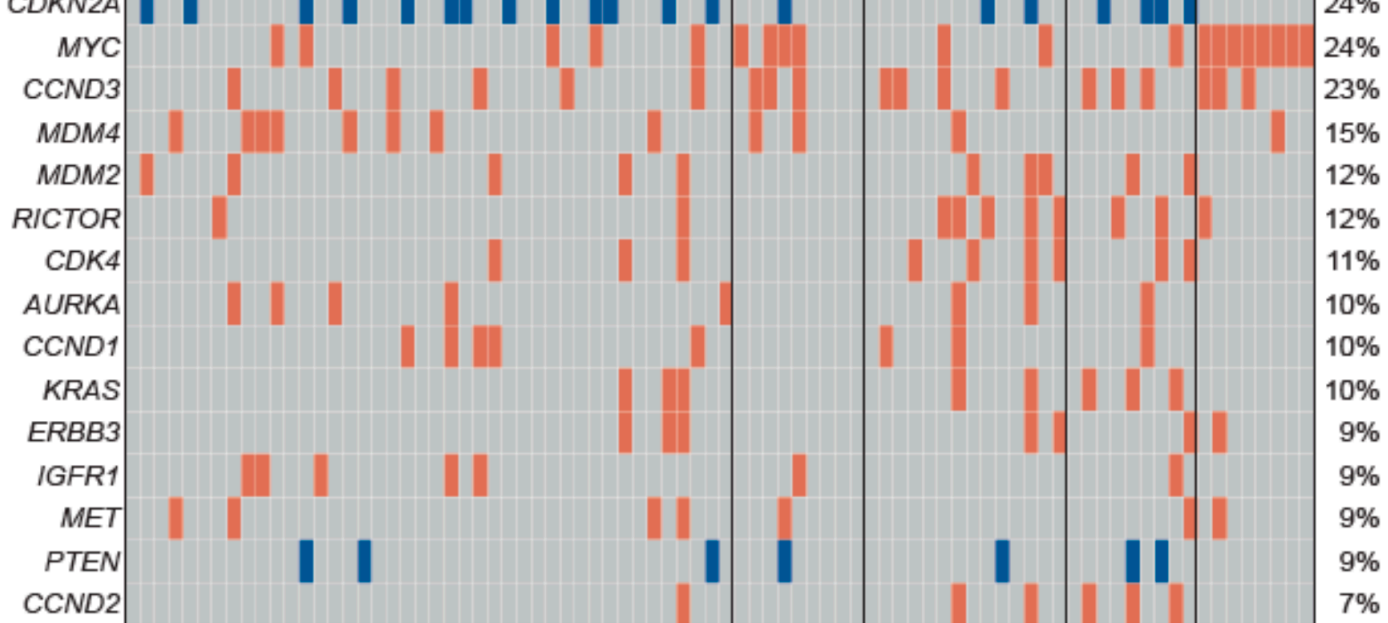

Variant classification

CNV classification

Melanoma subtype

Treatment

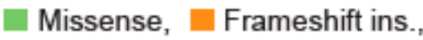

Splice site, Frameshift del.,

Amplification 24

口 cutaneous

occult

combi. Immunthx

Nonsense Inframe del.

acral-lentiginous

mucosal

uveal

Figure 1. Oncoplot of the most frequently mutated genes with driver mutations. Figure 1 shows a summary of the most frequent driver mutations of the melanoma cohort based on the 275 genes contained in all 3 panel versions. The Oncoplot shows the patients in a horizontal orientation and the gene and the corresponding driver mutations per patient in the vertical orientation. The plot is divided into three parts, in the upper area (a) all somatic single nucleotide variants (SNVs) and small insertions and deletions (INDELs) per patient, including the synonymous variants, are shown. The middle panel 
(b) summarizes all somatic SNVs found with a frequency of at least five percent, plus the two genes GNAQ and BAP1. The lower panel (c) summarizes the 15 most mutated genes with somatic copy number changes (CNVs). For both panel $(\mathbf{b})$ and (c), only mutations that were annotated as drivers or druggable biomarkers by the Cancer Genome Interpreter (CGI) are shown. Colors indicate different mutation types (s. legend for details). The entire cohort is sorted by the histopathological subtype in a grayscale (cutaneous, acral-lentiginous, mucosal, uveal, melanoma of unknown primary = occult). In addition, the type of therapy is annotated (red: combined immunotherapy, $n=60$; blue: anti-PD-1, $\mathrm{n}=15$; green: no immunotherapy, $\mathrm{n}=7$ ).

\subsection{Differences between Melanoma Subtypes}

As part of the further analysis, we attempted to identify characteristic genetic differences between the five histopathological melanoma subtypes, based on panel sequencing. Interestingly, we found great similarities between the cutaneous melanoma and melanoma of unknown primary. Both subtypes were dominated by somatic SNVs, leading to the highest number of mutations (median $=7$ ) and a significantly higher TMB (median $=9.4$, range $=0-36.4 ; p<0.003$ ) in patients with cutaneous melanoma compared to the other subtypes (Figure 2a, Table 2). The majority of the cutaneous and melanoma of unknown primary could be classified in the genomic subtypes BRAF, RAS and NF1. Only 19\% of cutaneous or 33\% melanoma of unknown primary were triple wild-type. In contrast, patients with acral and mucosal melanoma were triple wild-type in most of the cases (acral =72\%, mucosal = 78\%) and had CNVs predominantly. In patients with mucosal melanoma, we observed significantly more somatic CNVs (median $=33$, range $0-43 ; p<0.002$ ) than in cutaneous melanoma (Figure $2 b$, Table 2).

(a)

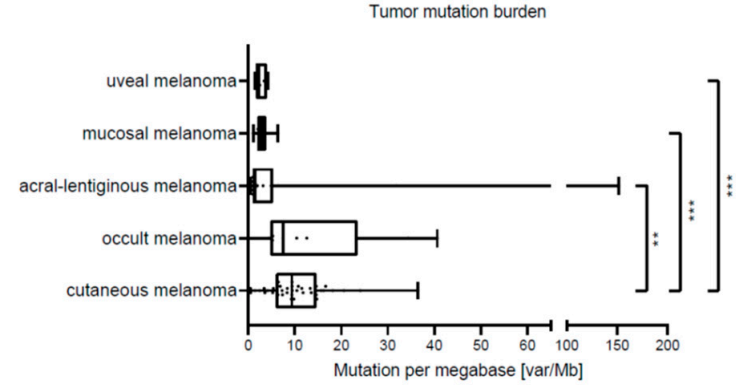

(c)

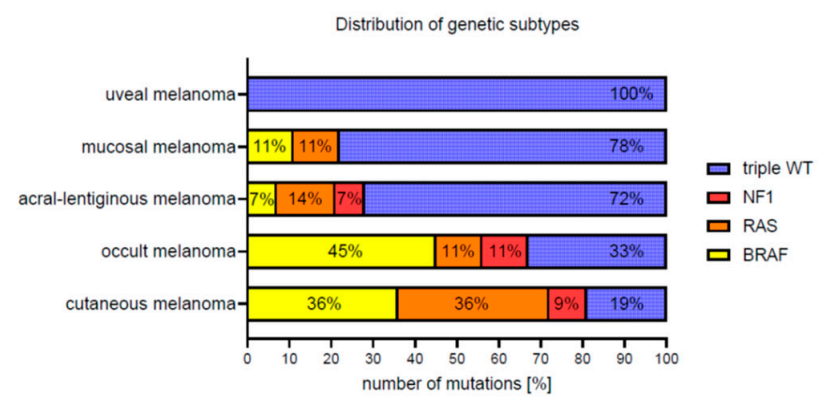

(b)

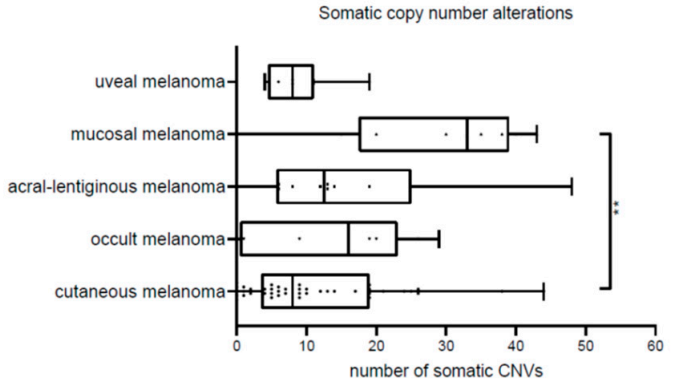

Figure 2. Tumor mutation burden, copy number variants and genetic subtypes. Figure 2 shows the comparison of tumor mutation burden (TMB), number of somatic copy number changes (CNVs) and the frequency of the genetic subtypes $B R A F, N R A S, N F 1$ and Triple wild-type (triple-WT) between the four histopathological subtypes (cutaneous, acral-lentiginous, mucosal, uveal) and the melanoma of unknown primary (occult). The figure consists of 3 histograms $(\mathbf{a}-\mathbf{c})$, whereby the y-axis always represents the histopathological subtypes. On the $\mathrm{x}$-axis the TMB is indicated in (a), as mutations per megabase (Mut/Mb), in (b) the number of somatic CNVs, in absolute numbers related to the 275 genes analyzed and in (c) the genetic subtype in absolute numbers. Patients with cutaneous melanoma have 
the most somatic SNVs and thus the highest median TMB compared to the remaining three subtypes $(p \leq 0.003)$. The median TMB of occult and cutaneous melanoma is only slightly different with $7.5 \mathrm{var} / \mathrm{Mb}$ versus $9.4 \mathrm{var} / \mathrm{Mb}$, respectively. The mucosal melanoma clearly has the most somatic CNVs with a median of 33 and there is a significant difference between the mucosal and cutaneous melanoma $(p=0.025)$ and the uveal and occult melanoma $(p=0.025)$. Cutaneous melanoma shows an even distribution of $B R A F$ and RAS mutated patients ( $40 \%$ each) and patients with melanoma of unknown primary are also in $45 \%$ BRAF mutated. The other three subtypes show a clear overweight of tumors with the status triple-WT.

To identify characteristic genetic differences between the histopathological melanoma subtypes, we performed a gene set enrichment analysis (Figure 3, Tables S10 and S11), revealing several significant differences. In contrast, uveal melanoma is the most distinct subtype. Compared to cutaneous, acral and mucosal melanoma, there was no enrichment of mutations in the RTK/RAS signaling pathway, but frequent mutually exclusive mutations in the genes of the guanine nucleotide-binding protein subunits GNA11 and GNAQ in combination with either the splicing factor SF3B1 or the deubiquitinating enzyme $B A P 1$ (Figure 3a,b). Of note, we found MYC amplifications in each of the eight uveal melanoma patients, which is a significant difference to the other melanoma subtypes $(p<0.05)$.

Table 2. Genetic characteristics of the 82 patients.

\begin{tabular}{|c|c|c|c|c|c|}
\hline $\begin{array}{l}\text { Genetic Characteristics of the } \\
82 \text { Patients }\end{array}$ & Cutaneous & Acral & Mucosal & Uveal & Occult \\
\hline \multicolumn{6}{|l|}{ Tumor mutation burden (TMB) } \\
\hline Range & $0-36.444$ & $0.51-151.8$ & $1.1-6 . \mathrm{a}$ & $1.5-4.2$ & $0-40.6$ \\
\hline Comparison to cutaneous subtype ${ }^{1}$ & & $p=0.0027 *$ & $p=0.0003^{*}$ & $p=0.0003^{*}$ & $p=0.837$ \\
\hline Median (IQR) & $10(6-15)$ & $3(1-7.3)$ & $3(2-4.5)$ & $3.5(2-4)$ & $11(4-25)$ \\
\hline Range & $0-33$ & $0-156$ & $1-6$ & $2-4$ & $1-44$ \\
\hline Comparison to cutaneous subtype ${ }^{1}$ & & $p=0.0134$ & $p=0.0005^{*}$ & $p=0.0008^{*}$ & $p=0.995$ \\
\hline \multicolumn{6}{|l|}{ Copy number variants (CNVs) } \\
\hline Median (IQR) & $8(3.5-19)$ & $12.5(5.8-25)$ & $33(17.5-39)$ & $8(4.5-11)$ & $16(0.5-23)$ \\
\hline
\end{tabular}

${ }^{1}$ Mann-Whitney UTest, ${ }^{*}$ significant.

On the other hand, hotspot mutations in the genes $B R A F$ and NRAS, in combination with homozygous deletions of the gene $C D K N 2 A$, were frequently found in cutaneous melanoma. In contrast, acral and mucosal melanoma were dominated by copy number changes affecting the RTK/RAS pathway and regulators of the cell cycle (Figure 3a). The co-amplifications of the receptor tyrosine kinases KIT and PDGFRA on chromosome 4, as well as CDK4 on chromosome 12, are of particular interest for acral melanoma. Mucosal melanoma had a significant accumulation of amplification in the regulator of the cell cycle control CCND2 (Figure 3b). Furthermore, a member of the Fanconi anemia pathway, CHEK2, was enriched for deletions in mucosal melanoma. 
(a)

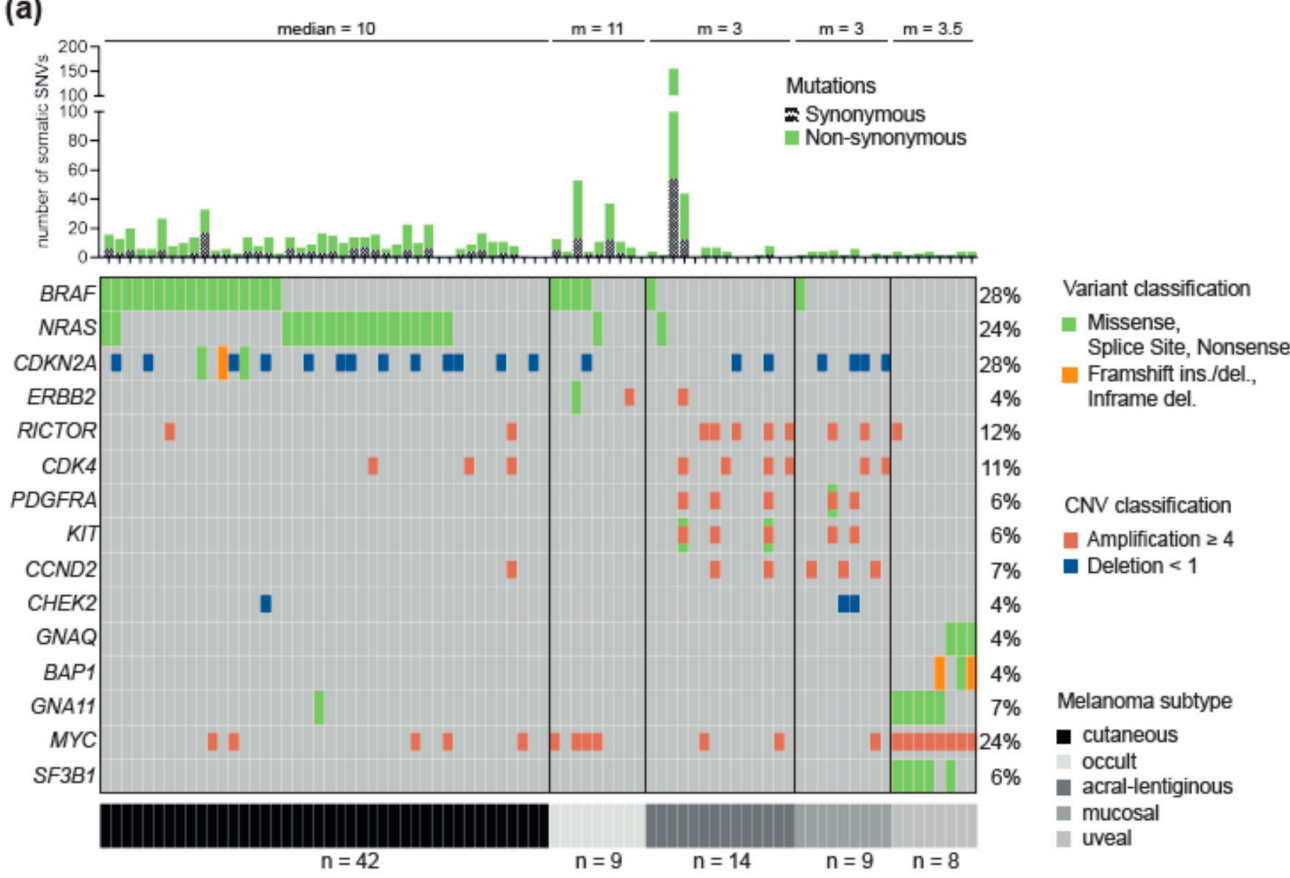

(b)

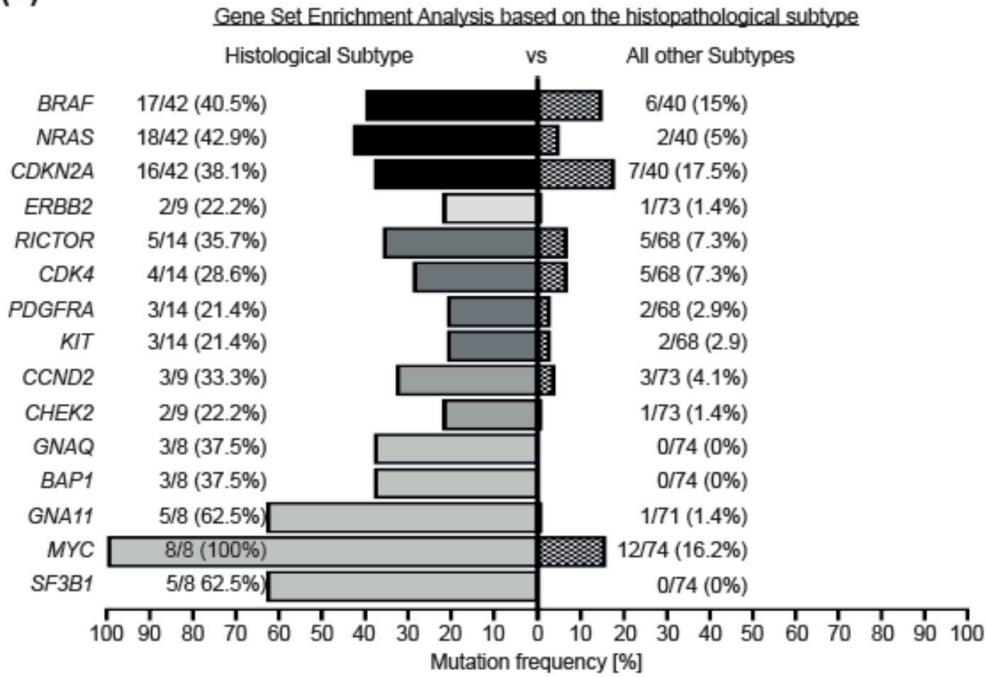

Figure 3. Gene set enrichment analysis of histopathological subtypes. Figure 3 summarizes the genetic differences, based on the gene set enrichment analysis (subtype versus rest of the cohort), of the four histopathological subtypes and the melanoma of unknown primary (occult). The figure consists of a histogram (a) showing the number of somatic changes (SNVs), including synonymous variants and the median (m) of each subtype. Below is an Oncoplot. It is sorted by histopathological subtypes cutaneous, acral-lentiginous, mucosal, occult and uveal melanoma (gray scale). It summarizes the genes that are significant $(p<0.05)$ for each subtype. In vertical alignment, the patients are summarized and in horizontal alignment the genes and the corresponding changes. On the left side the frequency, as a histogram in percent, of the somatic mutations (drivers) per gene, related to the total cohort, is shown. The panel (b) shows the frequency (in percent) of mutations per gene related to the subtypes (gray scale) compared to the rest of the cohort. For cutaneous melanoma, the genes BRAF $(p=0.009), N R A S$ $(p=0.0004)$ and $C D K N 2 A(p=0.03)$ are significantly enriched mutated compared to the rest of the cohort. For the acral-lentiginous melanoma the genes RICTOR $(p=0.01)$, CDK4 $(p=0.04)$, PDGFRA $(p=0.03)$ and KIT $(p=0.03)$ are enriched mutated, for the mucosal melanoma the genes CCND2 $(p=0.01)$ and CHEK2 $(p=0.03)$ are enriched mutated, for the melanoma of unknown primary the gene $\operatorname{ERBB2}(p=0.03)$ is enriched mutated and for the uveal melanoma the genes GNAQ ( $p=0.0006)$, GNA11 $(p=0.00001), B A P 1(p=0.0006), M Y C(p=0.000003)$ and SF3B1 $(p=0.0000002)$ are enriched mutated. 


\subsection{Characteristics of Patients Treated with Immune Checkpoint Inhibitors}

The majority of our cohort had been treated with ICI (75 of 82). Most of the patients $(n=60)$ had received combined immunotherapy with nivolumab and ipilimumab, and 15 patients had been treated only with PD-1 antibodies alone. In order to identify possible genes associated with resistance to ICI therapy, we classified treatment response according to RECIST 1.1 [24] in three groups: progressive disease (PD) $(n=45)$, stable disease (SD) $(n=12)$ and partial response $(P R)(n=18)$. Interestingly, most of the patients with PD and a BRAF mutation (16 of 18) had received therapy with BRAF and MEK inhibition (BRAF/MEKi) before initiation of ICI compared to those with stable disease or partial response ( 2 of 4 ) (Figure 4 ). As expected, the poorest survival was achieved by patients with PD, followed by patients with SD, while the best survival was that of patients with PR (Figure S1a). The one-year overall survival (OS) rate was $51 \%$ for patients with PD compared to $83 \%$ for SD and $100 \%$ for PR (Table S12).

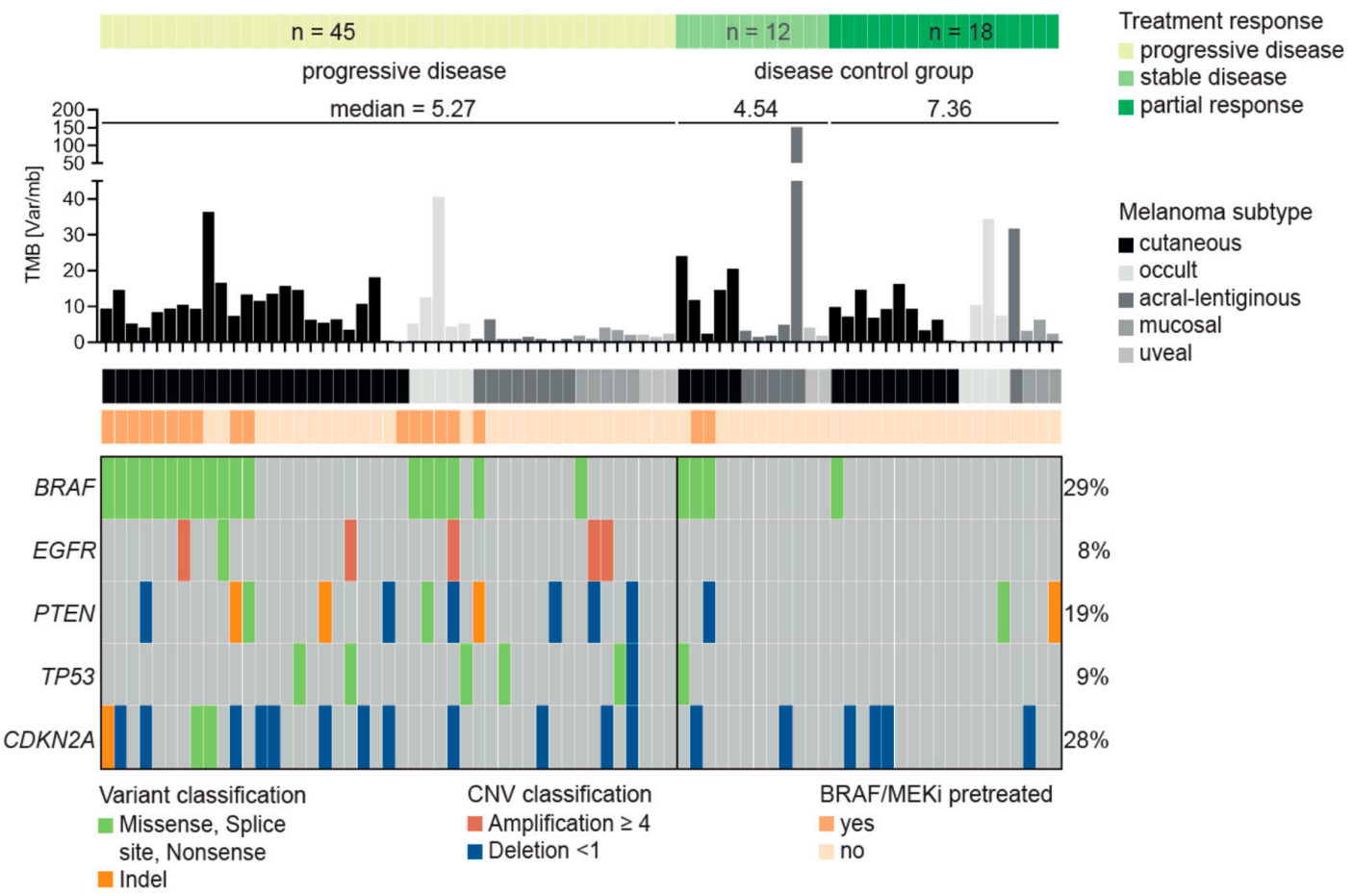

Figure 4. Predictor for resistance to immune checkpoint therapy. Figure 4 shows the result of the Fisher's test identifying a genetic predictor for the resistance to immune checkpoint inhibitor therapy based on the RECIST response classification (progressive disease versus disease control [stable disease, partial response]). Mutations in three genes-EGFR, PTEN and TP53 - were identified as individual predictors for a progressive disease at the first staging. In $89 \%$ of cases (16 out of 18 ), patients with $B R A F$ mutations had previously received BRAF/MEK inhibition before receiving immunotherapy. Together the mutations and the failed BRAF/MEKi pre-therapy predict $82 \%$ of the patients as PD $(p=0.007)$.

The comparison of the OS between the three groups of patients, i.e., (a) melanoma $B R A F$ wild-type, (b) $B R A F$ mutant (BRAF ${ }^{\mathrm{mut}}$ ) treatment naïve and (c) pretreated with $B R A F / M E K i$ (before ICI) melanoma showed a significantly better OS for patients in the first two groups $(p=0.01)$ (Figure S1b, Table S13). Similarly, the one-year OS rate was $80 \%$ for $B R A F-W T$ and $72 \%$ for treatment naïve $B R A F^{\text {mut }}$ patients compared to only $63 \%$ in patients who received prior therapy with a BRAF/MEKi.

\subsection{Resistance Predictor for Immune Checkpoint Inhibitor Therapy}

We next aimed at developing an ICI resistance predictor based on known resistance mechanisms. For instance, mutations in PTEN and amplifications of EGFR have previously been linked to resistance 
to ICI [25]. Mutations in TP53 have been associated with prolonged progression-free survival in lung cancer after immunotherapy [25], but also to increased ICI resistance in melanoma [26]. Indeed, we observed in our cohort that mutations in PTEN and EGFR are enriched in the PD group $(24.5 \%$ and $13.3 \%$ ) compared to the SD+PR group (10\% and $0 \%)$, although only the difference for EGFR is borderline significant. As previously suggested for melanoma, we also found that TP53 mutations are enriched in the PD (13.3\%) compared to the SD+PR group (3.3\%). As described above, we further observed that pretreatment with BRAF/MEKi is associated with PD $(p=0.01)$.

Combining these features in a multi-gene predictor of resistance to ICI, we observed that patients who are either pretreated with BRAF/MEKi or have a mutation in at least one of the genes PTEN, EGFR or TP53 are highly likely to show PD $(84.4 \%, p=0.0002$; Table S14). Accordingly, the relative risk (RR) to show PD in the first staging after starting with ICI was 1.99 for cases in which the tumor harbors a mutation in EGFR, PTEN or TP53 or the patient was pretreated with BRAF/MEKi. However, the sensitivity of the predictor is rather low, identifying $62 \%$ of PD patients (28/45), but with a good specificity of $80 \%$ (24/30). We also tested if a mutation in at least one of the genes PTEN, EGFR or TP53 alone (excluding BRAF/MEKi pretreatment as a feature) is a predictor of PD, which indeed is the case $(82 \%$ show PD, $p=0.007)$, although only $42 \%$ of the PD patients (19/45) are identified with this reduced combination $(\mathrm{RR}=1.65)$.

We have previously reported that deletions or loss of function mutations in CDKN2A increase the resistance to ICI [26]. Similarly, we find that $C D K N 2 A$ deletions or loss of heterozygosity are enriched in $\operatorname{PD}(p=0.024)$. However, mutations are mostly redundant to the already included genes, i.e., many patients with CDKN2A deletion also harbor a mutation in PTEN, EGFR or TP53. Still, the inclusion of $C D K N 2 A$ increases the sensitivity of predicting PD to $73.3 \%(33 / 45$ and $R R=2.05)$ but reduces specificity to $66.7 \%$ (20/30). Another well-known resistance gene, JAK2 [13,27], was only mutated in three patients, two of which additionally harbored a PTEN or an EGFR mutation. The highest specificity of $100 \%$ (30/30) is observed for any combination of the above changes in EGFR, PTEN, TP53 and CDKN2A. All nine patients with such a combination showed PD in the first staging, although the latter has reduced sensitivity ( $20 \%$ or $9 / 45)$. The absence of combined changes in these four genes could be associated with response to ICI, while the occurrence of such a combination seems to be highly predictive for a poor response $(\mathrm{RR}=1.83)$. The high number of unidentified progressions of ICI by this combined predictor indicates additional factors that influence ICI response.

\section{Discussion}

In this study, we aimed to evaluate TMB and driver mutations in a real-world cohort of patients with cutaneous, acral, mucosal and uveal melanoma and melanoma of unknown primary. Employing a targeted cancer panel approach, we found distinct genetic patterns for different histopathological subtypes. All subtypes were characterized by driver mutations in the genes of the RTK/RAS signaling pathway as well as cell cycle control and the PI3K pathway, except for patients with uveal melanoma. This subtype was dominated by amplification of MYC and GNA11 or GNAQ mutations in combination with the genes BAP1 or SF3B1. The high frequency of $M Y C$ amplification has been described in previous works, and it is known that high expression of $M Y C$ in tumors is associated with an increased risk of developing metastases and a worse prognosis [28-32]. Furthermore, we found mutual exclusivity of $S F 3 B 1$ or $B A P 1$ driver mutations in all patients with uveal melanoma. The splicing factor $S F 3 B 1$ is associated with an increased risk of metastasis and the deubiquitinase BAP1 with a worse prognosis [32,33]. These observations are in line with the clinical status of the included patients. All of them were already metastasized and characterized as advanced uveal melanomas.

The other three subtypes, as well as melanoma of unknown primary, were, as mentioned above, dominated by driver mutations in the genes of the cell cycle control $(C D K N 2 A)$ and the RTK/RAS signaling pathway (BRAF, NRAS, ERRB2). Besides, cutaneous and melanoma of unknown primary had the highest tumor mutation burden compared to the other subtypes. Interestingly, they are also similar in terms of the number of CNVs and the frequency of genomic subgroups, BRAF-, RAS-, NF1-mutated 
or triple wild type. Taken together, these results support the assumption that the cutaneous subtype and melanoma of unknown primary are similar and that the latter probably originates from regressed or unrecognized primary cutaneous melanomas [34,35].

In comparison, the number of somatic CNVs was higher in acral and mucosal melanoma than in cutaneous melanoma. This result corresponds to the current state of knowledge [36,37], whereby in our cohort, a significantly higher number of CNVs could only be detected in mucosal melanoma. The driver mutations in the genes RICTOR, CDK4, PDGFRA, KIT were specific for acral melanoma, while the amplification or deletion of the genes CCND2 and CHEK2 are uniquely found in mucosal melanoma. Our study confirms the observations reported in other recent studies [4-6].

Furthermore, the comparison of driver gene mutations in patients with PD compared to patients with SD+PR under ICI treatment revealed enrichment of EGFR, PTEN and TP53 gene mutations together with BRAF V600 mutations, if pretreated with BRAF/MEKi. We observed an $84.4 \%$ chance of progressive disease under ICI $(p=0.0002)$ for cases where the tumor harbors a mutation in one of these genes, or the patient was pretreated with BRAF/MEKi. Hence, the good performance of our multi-gene predictor supports previous observations and shows that the combined analysis of resistance mechanisms might be a powerful tool for a treatment recommendation. However, it is necessary to apply the predictor to an independent cohort in future studies to robustly evaluate its precision and recall. Moreover, other features such as TMB or additional genes could be incorporated to improve the predictor's sensitivity, although again, independent testing would be required. By combining similar studies, one might soon reach the critical mass to train machine learning based classifiers such as random forests, facilitating the inclusion of more features. Moreover, a large homogeneous cohort of a single melanoma subtype treated with ICI would very likely improve the accuracy of a trained predictor. Several studies in non-small-cell lung cancer have already shown the ineffectiveness of ICI in the case of tumors with EGFR mutations and low TMB [38-40]. Also, several retrospective studies have shown that the outcome of ICI is improved if these patients do not receive prior BRAF/MEKi therapy [41-43]. Moreover, an increased risk of progressive disease at the first staging was associated with the presence of PTEN and TP53 driver mutations. Both, if mutated, are potential genetic markers for resistance to ICI $[26,44]$.

Besides, driver mutations in the cyclin-dependent kinases $C D K N 2 A, C D K 4, C C N D 1 / 2 / 3$ and the genes MDM2/4 and TP53 were abundant, which presumably contribute to significant deregulation of cell cycle control. Deletions of gene CDKN2A appear to lead to resistance to immunotherapy and, in combination with the deregulation of $C D K 4$, worsen prognosis in acral melanoma [13,45]. Therefore, the introduction of $C D K 4 / 6$ inhibitors could broaden treatment options [45-47]. Combined treatment with $M E K$ and $C D K 4 / 6$ inhibitors could also be important [48]. Another potential treatment targets are driver mutations in the genes ATM, CHEK2, FANCA, FANCC and BRCA2, which belong to the DNA repair signaling pathway. One treatment option would be $P A R P$ inhibitors or platinum-based chemotherapy [49].

One of the limitations of the study is the fact that the evaluated cohort includes patients treated in daily clinical practice without further specific inclusion criteria. This means that all patients included were in an advanced stage of the disease, including distant metastases, and most of them had already received several systemic therapies. With regards to the differences between the melanoma subtypes $[50,51]$ and the identification of potential druggable targets, this is probably not that relevant. While there might be differences between primary melanoma and metastasis, it still seems to be appropriate to tailor the therapy regime to the genomic pattern of the metastases. Concerning the advanced tumor stages of the patients and prior systemic therapies, the interpretation of genetic patterns for ICI resistance might be difficult. Sequencing the tumor tissue at an earlier stage without pretreatment and at the time of progression might be more appropriate to detect possible genetic resistance mechanisms.

Finally, we observed that the TMB of cutaneous melanoma in our cohort was lower than that of TCGA [1]. This difference may be because the estimated TMB of our cohort is based on a panel of 
genes and, therefore, different from the whole-exome approach used in the TCGA cohort. Furthermore, we probably have a negative selection of patients in our cohort as most of our patients had not responded to ICI, which is likely to be more common when the TMB is lower [50-53].

\section{Conclusions}

NGS might help to characterize melanoma subtypes more precisely and to better understand ICI failure in some points. We observed that mutational signatures (TMB, CNV burden, driver genes) of melanoma of unknown primary resemble cutaneous melanomas. Furthermore, in patients with a progressive disease, we found a strong enrichment of cases with a mutation in at least one of the genes BRAF, EGFR, PTEN, TP53 or CDKN2A. Nevertheless, prospective studies are urgently needed to learn more about potential ICI resistance mechanisms, and further effort should be made to introduce targeted therapies beyond BRAF, MEK or KIT inhibitors, such as MYC or MDM2 inhibitors for metastasized melanoma.

Supplementary Materials: The following are available online at http://www.mdpi.com/2072-6694/12/9/2359/s1. Figure S1: Shown is the survival of the 75 patients who received immunocheckpoint therapy depending on response group (a) and the influence of BRAF mutation status and previous treatments (b). Table S1: Cancer panel version ssSCv2. Table S2: Cancer panel version ssSCv3. Table S3: Cancer panel version ssSCv4. Table S4: List of common 275 genes present in all three panel versions. Table S5: List of all somatic mutation (SNVs and INDELs) identified in all 82 patients unfiltered for the common 275 genes. Table S6: List of all somatic copy number variants (Amp \& Del) identified in all 82 patients unfiltered for the common 275 genes. Table S7: Tumor mutation burden [Mut/Mb] of all 82 patients. Table S8: List of all somatic mutation (SNVs and INDELs) filtered for the common 275 genes and driver mutations. Table S9: List of all somatic copy number variants (Amp \& Del) identified in all 82 patients filtered for the common 275 genes and driver mutations. Table S10: genomic subtype. Table S11: gene set enrichment based on histopathological subtype. Table S12: Overall survival based on disease groups. Table S13: Overall survival based on BRAF mutation status and pretreatment with BRAF/MEK inhibitors. Table S14: Fisher's test Immunotherapy Resistance Predictor.

Author Contributions: Conception and design: A.F., F.J.H., C.G., C.S. Development of methodology: A.F., F.J.H., G.D., S.O., C.S. Acquisition of data: A.F., F.J.H., S.O., C.S. Analysis and interpretation of data: all authors. Study supervision: A.F., C.G., C.S. Writing, review, revision of the manuscript: all authors. All authors have read and agreed the submitted version of the manuscript.

Funding: A.F. was supported by the TÜFF Habilitation Program for Women of the Faculty of Medicine Tübingen, Germany, grant no ${ }^{\circ}$ 2521-0-0. T.S. was supported by the Deutsche Forschungsgemeinschat (German Research Foundation) under Germany's Excellence Strategy_EXC 2180-390900677.

Acknowledgments: We thank the whole team of the melanoma outpatient department for their care for our melanoma patients.

Conflicts of Interest: A.F. served as consultant to Roche, Novartis, MSD, Pierre-Fabre; received travel support from Roche, Novartis, BMS, Pierre-Fabre, received speaker fees from Roche, Novartis, BMS, MSD and CeGaT, received research funding from BMS outside the submitted work. T.A. reports personal fees and travel grants from BMS, grants, personal fees and travel grants from Novartis, personal fees from Pierre Fabre and CeCaVa, grants from Neracare, grants from Sanofi, outside the submitted work. I.B. received speaker fees from Novartis and AstraZeneca and honoraria for advisory board participation from BMS and Novartis. CG reports grants and personal fees from Novartis, BMS, Roche, personal fees from MSD. Personal fees from Amgen, Philogen, LEO, Incyte, outside the submitted work. F.J.H., O.R. and C.S. received an institutional grant from Novartis. C.S. received research funding from BMS outside the submitted work. T.S. received grants from Novartis and Pierre-Fabre and personal fees from Neracare. The other authors declared no competing interests.

Data availability: The datasets used and/or analysed during the current study are available from the corresponding author on reasonable request.

\section{References}

1. Cancer Genome Atlas Network. Genomic Classification of Cutaneous Melanoma. Cell 2015, 161, 1681-1696. [CrossRef] [PubMed]

2. Krauthammer, M.; Kong, Y.; Bacchiocchi, A.; Evans, P.; Pornputtapong, N.; Wu, C.; McCusker, J.P.; Ma, S.; Cheng, E.; Straub, R.; et al. Exome sequencing identifies recurrent mutations in NF1 and RASopathy genes in sun-exposed melanomas. Nat. Genet. 2015, 47, 996-1002. [CrossRef] [PubMed] 
3. Hodis, E.; Watson, I.R.; Kryukov, G.V.; Arold, S.T.; Imielinski, M.; Theurillat, J.P.; Nickerson, E.; Auclair, D.; Li, L.; Place, C.; et al. A landscape of driver mutations in melanoma. Cell 2012, 150, 251-263. [CrossRef] [PubMed]

4. Hayward, N.K.; Wilmott, J.S.; Waddell, N.; Johansson, P.A.; Field, M.A.; Nones, K.; Patch, A.M.; Kakavand, H.; Alexandrov, L.B.; Burke, H.; et al. Whole-genome landscapes of major melanoma subtypes. Nature 2017, 545, 175-180. [CrossRef]

5. $\quad$ Liang, W.S.; Hendricks, W.; Kiefer, J.; Schmidt, J.; Sekar, S.; Carpten, J.; Craig, D.W.; Adkins, J.; Cuyugan, L.; Manojlovic, Z.; et al. Integrated genomic analyses reveal frequent TERT aberrations in acral melanoma. Genome Res. 2017, 27, 524-532. [CrossRef]

6. Newell, F.; Kong, Y.; Wilmott, J.S.; Johansson, P.A.; Ferguson, P.M.; Cui, C.; Li, Z.; Kazakoff, S.H.; Burke, H.; Dodds, T.J.; et al. Whole-genome landscape of mucosal melanoma reveals diverse drivers and therapeutic targets. Nat. Commun. 2019, 10, 3163. [CrossRef]

7. Garman, B.; Anastopoulos, I.N.; Krepler, C.; Brafford, P.; Sproesser, K.; Jiang, Y.; Wubbenhorst, B.; Amaravadi, R.; Bennett, J.; Beqiri, M.; et al. Genetic and Genomic Characterization of 462 Melanoma Patient-Derived Xenografts, Tumor Biopsies, and Cell Lines. Cell Rep. 2017, 21, 1936-1952. [CrossRef]

8. Krepler, C.; Sproesser, K.; Brafford, P.; Beqiri, M.; Garman, B.; Xiao, M.; Shannan, B.; Watters, A.; Perego, M.; Zhang, G.; et al. A Comprehensive Patient-Derived Xenograft Collection Representing the Heterogeneity of Melanoma. Cell Rep. 2017, 21, 1953-1967. [CrossRef]

9. Kato, S.; Goodman, A.; Walavalkar, V.; Barkauskas, D.A.; Sharabi, A.; Kurzrock, R. Hyperprogressors after Immunotherapy: Analysis of Genomic Alterations Associated with Accelerated Growth Rate. Clin. Cancer Res. 2017, 23, 4242-4250. [CrossRef]

10. Forschner, A.; Niessner, H.; Moller, Y.; Horak, P.; Frohlich, M.; Warsow, G.; Stenzinger, A.; Frohling, S.; Glimm, H.; Klumpp, B.; et al. Genomics of Immunotherapy-Associated Hyperprogressors-Letter. Clin. Cancer Res. 2017, 23, 6374-6375. [CrossRef]

11. Forschner, A.; Hilke, F.J.; Bonzheim, I.; Gschwind, A.; Demidov, G.; Amaral, T.; Ossowski, S.; Riess, O.; Schroeder, C.; Martus, P.; et al. MDM2, MDM4 and EGFR Amplifications and Hyperprogression in Metastatic Acral and Mucosal Melanoma. Cancers 2020, 12, 540. [CrossRef] [PubMed]

12. Trujillo, J.A.; Luke, J.J.; Zha, Y.; Segal, J.P.; Ritterhouse, L.L.; Spranger, S.; Matijevich, K.; Gajewski, T.F. Secondary resistance to immunotherapy associated with beta-catenin pathway activation or PTEN loss in metastatic melanoma. J. Immunother. Cancer 2019, 7, 295. [CrossRef] [PubMed]

13. Horn, S.; Leonardelli, S.; Sucker, A.; Schadendorf, D.; Griewank, K.G.; Paschen, A. Tumor CDKN2A-Associated JAK2 Loss and Susceptibility to Immunotherapy Resistance. J. Natl. Cancer Inst. 2018, 110, 677-681. [CrossRef] [PubMed]

14. Zaretsky, J.M.; Garcia-Diaz, A.; Shin, D.S.; Escuin-Ordinas, H.; Hugo, W.; Hu-Lieskovan, S.; Torrejon, D.Y.; Abril-Rodriguez, G.; Sandoval, S.; Barthly, L.; et al. Mutations Associated with Acquired Resistance to PD-1 Blockade in Melanoma. N. Engl. J. Med. 2016, 375, 819-829. [CrossRef] [PubMed]

15. Gao, J.; Shi, L.Z.; Zhao, H.; Chen, J.; Xiong, L.; He, Q.; Chen, T.; Roszik, J.; Bernatchez, C.; Woodman, S.E.; et al. Loss of IFN-gamma Pathway Genes in Tumor Cells as a Mechanism of Resistance to Anti-CTLA-4 Therapy. Cell 2016, 167, 397-404. [CrossRef]

16. Boshuizen, J.; Koopman, L.A.; Krijgsman, O.; Shahrabi, A.; van den Heuvel, E.G.; Ligtenberg, M.A.; Vredevoogd, D.W.; Kemper, K.; Kuilman, T.; Song, J.Y.; et al. Cooperative targeting of melanoma heterogeneity with an AXL antibody-drug conjugate and BRAF/MEK inhibitors. Nat. Med. 2018, 24, 203-212. [CrossRef]

17. Li, H.; Durbin, R. Fast and accurate short read alignment with Burrows-Wheeler transform. Bioinformatics 2009, 25, 1754-1760. [CrossRef]

18. Kim, S.; Scheffler, K.; Halpern, A.L.; Bekritsky, M.A.; Noh, E.; Kallberg, M.; Chen, X.; Kim, Y.; Beyter, D.; Krusche, P.; et al. Strelka2: Fast and accurate calling of germline and somatic variants. Nat. Methods 2018, 15, 591-594. [CrossRef]

19. Demidov, G.; Ossowski, S. ClinCNV: Novel method for allele-specific somatic copy-number alterations detection. bioRxiv 2019. [CrossRef]

20. Cingolani, P.; Platts, A.; Wang le, L.; Coon, M.; Nguyen, T.; Wang, L.; Land, S.J.; Lu, X.; Ruden, D.M. A program for annotating and predicting the effects of single nucleotide polymorphisms, SnpEff: SNPs in the genome of Drosophila melanogaster strain w1118; iso-2; iso-3. Fly 2012, 6, 80-92. [CrossRef] 
21. Cingolani, P.; Patel, V.M.; Coon, M.; Nguyen, T.; Land, S.J.; Ruden, D.M.; Lu, X. Using Drosophila melanogaster as a Model for Genotoxic Chemical Mutational Studies with a New Program, SnpSift. Front. Genet. 2012, 3, 35. [CrossRef] [PubMed]

22. Tamborero, D.; Rubio-Perez, C.; Deu-Pons, J.; Schroeder, M.P.; Vivancos, A.; Rovira, A.; Tusquets, I.; Albanell, J.; Rodon, J.; Tabernero, J.; et al. Cancer Genome Interpreter annotates the biological and clinical relevance of tumor alterations. Genome Med. 2018, 10, 25. [CrossRef] [PubMed]

23. Mayakonda, A.; Lin, D.C.; Assenov, Y.; Plass, C.; Koeffler, H.P. Maftools: Efficient and comprehensive analysis of somatic variants in cancer. Genome Res. 2018, 28, 1747-1756. [CrossRef] [PubMed]

24. Eisenhauer, E.A.; Therasse, P.; Bogaerts, J.; Schwartz, L.H.; Sargent, D.; Ford, R.; Dancey, J.; Arbuck, S.; Gwyther, S.; Mooney, M.; et al. New response evaluation criteria in solid tumours: Revised RECIST guideline (version 1.1). Eur. J. Cancer 2009, 45, 228-247. [CrossRef] [PubMed]

25. Assoun, S.; Theou-Anton, N.; Nguenang, M.; Cazes, A.; Danel, C.; Abbar, B.; Pluvy, J.; Gounant, V.; Khalil, A.; Namour, C.; et al. Association of TP53 mutations with response and longer survival under immune checkpoint inhibitors in advanced non-small-cell lung cancer. Lung Cancer 2019, 132, 65-71. [CrossRef]

26. Brenner, E.; Schorg, B.F.; Ahmetlic, F.; Wieder, T.; Hilke, F.J.; Simon, N.; Schroeder, C.; Demidov, G.; Riedel, T.; Fehrenbacher, B.; et al. Cancer immune control needs senescence induction by interferon-dependent cell cycle regulator pathways in tumours. Nat. Commun. 2020, 11, 1335. [CrossRef]

27. Ayers, M.; Lunceford, J.; Nebozhyn, M.; Murphy, E.; Loboda, A.; Kaufman, D.R.; Albright, A.; Cheng, J.D.; Kang, S.P.; Shankaran, V.; et al. IFN-gamma-related mRNA profile predicts clinical response to PD-1 blockade. J. Clin. Investig. 2017, 127, 2930-2940. [CrossRef]

28. Parrella, P.; Caballero, O.L.; Sidransky, D.; Merbs, S.L. Detection of c-myc amplification in uveal melanoma by fluorescent in situ hybridization. Investig. Ophthalmol. Vis. Sci. 2001, 42, 1679-1684.

29. Lin, X.; Sun, R.; Zhao, X.; Zhu, D.; Zhao, X.; Gu, Q.; Dong, X.; Zhang, D.; Zhang, Y.; Li, Y.; et al. C-myc overexpression drives melanoma metastasis by promoting vasculogenic mimicry via c-myc/snail/Bax signaling. J. Mol. Med. 2017, 95, 53-67. [CrossRef]

30. Wolfer, A.; Ramaswamy, S. MYC and metastasis. Cancer Res. 2011, 71, 2034-2037. [CrossRef]

31. McCarthy, C.; Kalirai, H.; Lake, S.L.; Dodson, A.; Damato, B.E.; Coupland, S.E. Insights into genetic alterations of liver metastases from uveal melanoma. Pigment Cell Melanoma Res. 2016, 29, 60-67. [CrossRef] [PubMed]

32. Robertson, A.G.; Shih, J.; Yau, C.; Gibb, E.A.; Oba, J.; Mungall, K.L.; Hess, J.M.; Uzunangelov, V.; Walter, V.; Danilova, L.; et al. Integrative Analysis Identifies Four Molecular and Clinical Subsets in Uveal Melanoma. Cancer Cell 2017, 32, 204-220. [CrossRef] [PubMed]

33. Park, J.J.; Diefenbach, R.J.; Joshua, A.M.; Kefford, R.F.; Carlino, M.S.; Rizos, H. Oncogenic signaling in uveal melanoma. Pigment Cell Melanoma Res. 2018, 31, 661-672. [CrossRef] [PubMed]

34. Egberts, F.; Bergner, I.; Kruger, S.; Haag, J.; Behrens, H.M.; Hauschild, A.; Rocken, C. Metastatic melanoma of unknown primary resembles the genotype of cutaneous melanomas. Ann. Oncol. 2014, 25, 246-250. [CrossRef]

35. Heppt, M.V.; Tietze, J.K.; Reinholz, M.; Rahimi, F.; Jung, A.; Kirchner, T.; Ruzicka, T.; Flaig, M.J.; Berking, C. Disease kinetics but not disease burden is relevant for survival in melanoma of unknown primary tumor. Discov. Med. 2015, 20, 231-237.

36. Curtin, J.A.; Fridlyand, J.; Kageshita, T.; Patel, H.N.; Busam, K.J.; Kutzner, H.; Cho, K.H.; Aiba, S.; Brocker, E.B.; LeBoit, P.E.; et al. Distinct sets of genetic alterations in melanoma. N. Engl. J. Med. 2005, 353, 2135-2147. [CrossRef]

37. Furney, S.J.; Pedersen, M.; Gentien, D.; Dumont, A.G.; Rapinat, A.; Desjardins, L.; Turajlic, S.; Piperno-Neumann, S.; de la Grange, P.; Roman-Roman, S.; et al. SF3B1 mutations are associated with alternative splicing in uveal melanoma. Cancer Discov. 2013, 3, 1122-1129. [CrossRef]

38. Fruh, M.; Peters, S. EGFR mutation subtype impacts efficacy of immune checkpoint inhibitors in non-small-cell lung cancer. Ann. Oncol. 2019, 30, 1190-1192. [CrossRef]

39. Lee, C.K.; Man, J.; Lord, S.; Links, M.; Gebski, V.; Mok, T.; Yang, J.C. Checkpoint Inhibitors in Metastatic EGFR-Mutated Non-Small Cell Lung Cancer-A Meta-Analysis. J. Thorac. Oncol. 2017, 12, 403-407. [CrossRef]

40. Ozaki, Y.; Muto, S.; Takagi, H.; Watanabe, M.; Inoue, T.; Fukuhara, M.; Yamaura, T.; Okabe, N.; Matsumura, Y.; Hasegawa, T.; et al. Tumor mutation burden and immunological, genomic, and clinicopathological factors as biomarkers for checkpoint inhibitor treatment of patients with non-small-cell lung cancer. Cancer Immunol. Immunother. 2020, 69, 127-134. [CrossRef] 
41. Johnson, D.B.; Pectasides, E.; Feld, E.; Ye, F.; Zhao, S.; Johnpulle, R.; Merritt, R.; McDermott, D.F.; Puzanov, I.; Lawrence, D.; et al. Sequencing Treatment in BRAFV600 Mutant Melanoma: Anti-PD-1 Before and After BRAF Inhibition. J. Immunother. 2017, 40, 31-35. [CrossRef]

42. Moser, J.C.; Chen, D.; Hu-Lieskovan, S.; Grossmann, K.F.; Patel, S.; Colonna, S.V.; Ying, J.; Hyngstrom, J.R. Real-world survival of patients with advanced BRAF V600 mutated melanoma treated with front-line BRAF/MEK inhibitors, anti-PD-1 antibodies, or nivolumab/ipilimumab. Cancer Med. 2019, 8, 7637-7643. [CrossRef] [PubMed]

43. Schilling, B.; Martens, A.; Geukes Foppen, M.H.; Gebhardt, C.; Hassel, J.C.; Rozeman, E.A.; Gesierich, A.; Gutzmer, R.; Kahler, K.C.; Livingstone, E.; et al. First-line therapy-stratified survival in BRAF-mutant melanoma: A retrospective multicenter analysis. Cancer. Immunol. Immunother. 2019, 68, 765-772. [CrossRef] [PubMed]

44. Fares, C.M.; Van Allen, E.M.; Drake, C.G.; Allison, J.P.; Hu-Lieskovan, S. Mechanisms of Resistance to Immune Checkpoint Blockade: Why Does Checkpoint Inhibitor Immunotherapy Not Work for All Patients? Am. Soc. Clin. Oncol. Educ. Book/ASCO Am. Soc. Clin. Oncol. Educ. Meet. 2019, 39, 147-164. [CrossRef]

45. Kong, Y.; Sheng, X.; Wu, X.; Yan, J.; Ma, M.; Yu, J.; Si, L.; Chi, Z.; Cui, C.; Dai, J.; et al. Frequent Genetic Aberrations in the CDK4 Pathway in Acral Melanoma indicate the potential for CDK4/6 Inhibitors in Targeted Therapy. Clin. Cancer Res. 2017, 23, 6946-6957. [CrossRef] [PubMed]

46. Tang, B.; Sheng, X.; Kong, Y.; Chi, Z.; Si, L.; Cui, C.; Yan, X.; Mao, L.; Lian, B.; Li, S.; et al. Palbociclib for treatment of metastatic melanoma with copy number variations of CDK4 pathway: Case report. Chin. Clin. Oncol. 2018. [CrossRef]

47. Young, R.J.; Waldeck, K.; Martin, C.; Foo, J.H.; Cameron, D.P.; Kirby, L.; Do, H.; Mitchell, C.; Cullinane, C.; Liu, W.; et al. Loss of CDKN2A expression is a frequent event in primary invasive melanoma and correlates with sensitivity to the CDK4/6 inhibitor PD0332991 in melanoma cell lines. Pigment Cell Melanoma Res. 2014, 27, 590-600. [CrossRef]

48. Posch, C.; Sanlorenzo, M.; Ma, J.; Kim, S.T.; Zekhtser, M.; Ortiz-Urda, S. MEK/CDK4,6 co-targeting is effective in a subset of NRAS, BRAF and 'wild type' melanomas. Oncotarget 2018, 9, 34990-34995. [CrossRef]

49. Mateo, J.; Lord, C.J.; Serra, V.; Tutt, A.; Balmana, J.; Castroviejo-Bermejo, M.; Cruz, C.; Oaknin, A.; Kaye, S.B.; de Bono, J.S. A decade of clinical development of PARP inhibitors in perspective. Ann. Oncol. 2019, 30, 1437-1447. [CrossRef]

50. Van Allen, E.M.; Miao, D.; Schilling, B.; Shukla, S.A.; Blank, C.; Zimmer, L.; Sucker, A.; Hillen, U.; Foppen, M.H.G.; Goldinger, S.M.; et al. Genomic correlates of response to CTLA-4 blockade in metastatic melanoma. Science 2015, 350, 207-211. [CrossRef]

51. Johnson, D.B.; Frampton, G.M.; Rioth, M.J.; Yusko, E.; Xu, Y.; Guo, X.; Ennis, R.C.; Fabrizio, D.; Chalmers, Z.R.; Greenbowe, J.; et al. Targeted Next Generation Sequencing Identifies Markers of Response to PD-1 Blockade. Cancer Immunol. Res. 2016, 4, 959-967. [CrossRef] [PubMed]

52. Snyder, A.; Makarov, V.; Merghoub, T.; Yuan, J.; Zaretsky, J.M.; Desrichard, A.; Walsh, L.A.; Postow, M.A.; Wong, P.; Ho, T.S.; et al. Genetic basis for clinical response to CTLA-4 blockade in melanoma. N. Engl. J. Med. 2014, 371, 2189-2199. [CrossRef] [PubMed]

53. Forschner, A.; Battke, F.; Hadaschik, D.; Schulze, M.; Weissgraeber, S.; Han, C.T.; Kopp, M.; Frick, M.; Klumpp, B.; Tietze, N.; et al. Tumor mutation burden and circulating tumor DNA in combined CTLA-4 and PD-1 antibody therapy in metastatic melanoma-results of a prospective biomarker study. J. Immunother. Cancer 2019, 7, 180. [CrossRef] [PubMed]

(C) 2020 by the authors. Licensee MDPI, Basel, Switzerland. This article is an open access article distributed under the terms and conditions of the Creative Commons Attribution (CC BY) license (http://creativecommons.org/licenses/by/4.0/). 